\title{
EXPERIENCIA CON MONITOREO AMBULATORIO DE PRESIÓN ARTERIAL DE 24 HORAS EN SEGUIMIENTO DE PACIENTES CON COARTACIÓN DE AORTA EN UN HOSPITAL PEDIÁTRICO
}

\section{EXPERIENCE WITH 24-HOUR AMBULATORY BLOOD PRESSURE MONITORING IN FOLLOW-UP OF PATIENTS WITH AORTIC COARTACION IN A PEDIATRIC HOSPITAL \\ EXPERIÊNCIA COM MONITORAMENTO AMBULATORIAL DA PRESSÃO ARTERIAL POR 24 HORAS NO ACOMPANHAMENTO DE PACIENTES COM COARTAÇÃO AÓRTICA EM UM HOSPITAL PEDIÁTRICO}

\author{
Marina Vaccari ${ }^{1-2}$, Maria E. Molina ${ }^{1}$, Adriana Iturzaeta ${ }^{1}$, Fernando A. Torres ${ }^{1}$, Ines A. Martinez ${ }^{1}$, Judith B. Ackerman ${ }^{1}$, José $^{\prime}$ \\ E. Flores ${ }^{1}$, Jesus M. Damsky Barbosa ${ }^{1}$.
}

Los pacientes coartados operados necesitan tener control de la presión arterial durante toda su vida, ya que por su enfermedad de base tienen mayor riesgo de sufrir hipertensión arterial y eventos cardivasculres que la población gener El estudio de monitoreo ambulatorio do presín arterial (MAPA) o presurometrí de 24 horas permite el correcto seguimiento en estos evios car in les mostramos nuestros resultados.

\section{Conceptos clave:}

Los pacientes con antecedentes de coartación de aorta tienen mayor prevalencia de hipertensión arterial (HTA) a largo plazo, con más morbimortalidad que la población general.

El estudio de la HTA incluye la realización de monitoreo ambulatorio de presión arterial (MAPA). La detección de HTA por MAPA y el diagnóstico de HTA enmascarada conlleva un mayor riesgo cardiovascular y define cambios terapéuticos.

El trabajo se propuso analizar los cambios en la clasificación y el manejo de la HTA después de la realización de MAPA en pacientes pediátricos con antecedentes de coartación de aorta seguidos en un servicio de Cardiología Infantil.

Hospital de Niños Pedro Elizalde

2- E-mail de contacto: marinvaccari@yahoo.com.ar

Recibido: 2019-11-22 Aceptado: 2020-05-07

DOI: http://dx.doi.org/10.31053/1853.0605.v77.n4.26509

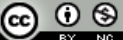

(c) Universidad Nacional de Córdoba

\section{Resumen:}

Introducción: La hipertensión arterial (HTA) es una comorbilidad importante en niños con coartación de aorta (COAO) y el monitoreo ambulatorio de presión arterial de 24horas (MAPA) permite un diagnóstico preciso.

Objetivo: Describir la prevalencia de HTA por presión arterial (PA) en consultorio y su recategorización con MAPA

Material y método: Estudio descriptivo, observacional, retrospectivo; incluyó niños entre 4y18 años con COAO que realizaron MAPA. Se registró PA en consultorio y MAPA, ecocardiograma y medicación.

Resultados: 33 pacientes, 26 varones, edad 10,2 $\pm 3,8$ años, Por PA en consultorio: 22 normotensos; 8 HTA controlada; 2 preHTA; 1 HTA no medicado. Con 32 registros completos de MAPA, se recategorizaron: normotensos 11, preHTA 7, HTA nocturna 3 , HTA enmascarada 4; HTA controlada 3; HTA no controlada 3 y 1 HTA.

Conclusión: La prevalencia de HTA en esta población en consultorio fue baja. EI MAPA recategorizó y detectó HTA nocturna e HTA enmascarada.

Palabras clave: hipertensión; hipertensión enmascarada; coartación aórtica; monitoreo ambulatorio de la presión arterial.

\section{Abstract:}

Introduction: Hypertension (HTA) is an important comorbidity in children with aortic coarctation (COAO) and 24-hour ambulatory blood pressure monitoring (ABPM) allows an accurate diagnosis.

Objective: Describe the prevalence of HTA in the office and its recategorization with ABPM

Material and method: Descriptive, observational, retrospective study; It included children between 4 and 18 years with COAO who performed ABPM. PA was registered in the office and ABPM, echocardiogram and medication.

Results: 33 patients, 26 men, age $10.2 \pm 3.8$ years, By PA in the office: 22 normotensive; 8 HTA controlled; 2 preHTA; 1 HTA not medicated. With 32 complete MAP records, they were categorized: normotensive 11, preHTA 7, nocturnal HTA 3, masked HTA 4; HTA controlled 3; Uncontrolled HTA 3 and 1 HTA.

Conclusion: The prevalence of hypertension in this population in the office was low. The ABPM recategorized and detected nocturnal HTA and masked HTA.

Keywords: hypertension; masked hypertension; coarctation of aorta; ambulatory blood pressure monitoring.

\section{Resumo}

Introdução: A hipertensão arterial (AHT) é uma comorbidade importante em crianças com coarctação de aorta (COAO) e o monitoramento ambulatorial de pressão arterial de 24 horas (MAPA) permite um diagnóstico preciso.

Objetivo: Descrever a prevalência de hipertensão por pressão arterial (PA) no consultório e sua recategorização na MAPA.

Material e método: Estudo descritivo, observacional, retrospectivo; Incluiu crianças entre 4 e 18 anos com COAO que realizaram PAM. A AP foi registrada no consultório e MAPA, ecocardiograma e medicação.

Resultados: 33 pacientes, 26 homens, idade 10,2 $\pm 3,8$ anos, por PA no escritório: 22 normotensos; 8 HTA controlado; 2 preHTA; 1 HTA não medicado. Com 32 registros completos do MAP, foram recategorizados: normotensos 11, pré-HTA 7, HTA 3 noturno, HTA 4 mascarado; Controlado por HTA 3; HTA 3 não controlado e 1 HTA.

Conclusão: A prevalência de hipertensão nessa população no consultório foi baixa. $O$ MAP recategorizou e detectou HTA noturno e HTA mascarado.

Palavras-chave: hipertensão; hipertensão mascarada; coarctação aórtica; monitorização ambulatorial da pressão arterial. 


\section{Introducción}

La coartación de aorta (COAO) es una cardiopatía congénita con una frecuencia de $5 \%$ a $8 \%{ }^{(1)}$. Puede ocurrir aislada o asociada a anomalías cardiacas más complejas ${ }^{(2)}$. Se define como un estrechamiento significativo del arco aórtico a nivel del istmo. Se considera un síndrome cardiovascular que incluye vasculopatía y enfermedad cerebrovascular ${ }^{(3)}$.

La hipertensión arterial (HTA) durante la infancia es la comorbilidad más importante asociada a COAO, antes y después de la reparación ${ }^{(1)}$. La prevalencia reportada de HTA en el seguimiento es del $25 \%$ al $68 \%{ }^{(3)}$.

La COAO se asocia a una menor esperanza de vida, principalmente debido a los efectos de la HTA crónica, con frecuencia refractaria ${ }^{(4-6)}$.

Para el seguimiento, varios autores sugieren la evaluación con monitoreo domiciliario de presión arterial de 24 horas (MAPA) como control más sensible de la presión arterial $(P A)^{(7-9)}$. Y es solo mediante el MAPA que se diagnostica Hipertensión Enmascarada (HE) e Hipertensión Arterial Nocturna (HTA nocturna) ${ }^{(10-11)}$.

Este estudio pretende describir la experiencia de un hospital pediátrico en la evaluación y manejo de pacientes con COAO luego de la incorporación sistemática de MAPA.

\section{Objetivos}

Describir la prevalencia de HTA en niños con COAO por PA en consultorio y la necesidad de recategorizar el diagnóstico luego de realizar MAPA.

\section{Material y Métodos}

Estudio retrospectivo, descriptivo, observacional. Se incluyeron todos los pacientes con COAO seguidos en los servicios de Cardiología e Pediatría del Hospital de Niños Pedro Elizalde a los que se le realizó MAPA entre 2017 y 2018. Se excluyeron registros clínicos incompletos y asociaciones sindrómicas.

Se registraron edad, sexo, peso y talla.

\section{Presión Arterial en consultorio (PAc)}

Registrada por método auscultatorio y analizada según género, edad y talla, de acuerdo a Tablas internacionales, con valores posibles ${ }^{(10)}$ :

1. Normotensión (< percentilo (PC) 90);

2. Prehipertensión ( $\mathrm{PC} \geq 90 \mathrm{Y} P C<95)$;

3. $\mathrm{HTA}(\geq \mathrm{PC} 95$ en pacientes que no reciben medicación antihipertensiva);

4. HTA controlada(< PC 95 en paciente que recibe medicación antihipertensiva);

5. HTA no controlada ( $P C \geq 95$, en paciente que recibe medicación antihipertensiva)

Monitoreo Ambulatorio de Presión Arterial (MAPA) Realizado por Spacelabs $90217^{a}$ y analizado según género y talla, de acuerdo a normas internacionales con valores posibles:

1. Normotenso: promedio PAS y/o PAD ambulatoria diurna y nocturna < PC 95.
2. HTA enmascarada: promedio PAS y/o PAD ambulatoria diurno > PC 95 con PAc normal.

3. HTA de guardapolvo blanco: promedio de PAS y/o PAD ambulatoria < PC 95, con PAc $\geq P C 95$.

4. Prehipertensión: promedio de PAS y/o PAD ambulatoria < PC 95, con más del $25 \%$ de carga.

5. HTA nocturna: promedio PAS y/o PAD ambulatoria nocturno >PC 95.

6. HTA controlada: promedio PAS y PAD ambulatoria diurna y nocturna $<P C 95$ en paciente que recibe medicación antihipertensiva.

7. HTA no controlada: promedio PAS y PAD ambulatoria diurna y nocturna > PC 95 en paciente que recibe medicación antihipertensiva.

8. HTA: promedio PAS y PAD ambulatoria diurna y nocturna > PC 95 en paciente que no recibe medicación antihipertensiva.

\section{Ecocardiograma 2D y Doppler color}

1. Gradiente en aorta descendente: desde toma supraesternal, se registró el gradiente máximo en $\mathrm{mmHg}$.

2. Pulsatilidad en aorta abdominal: desde toma subxifoidea, definida normal por flujo pulsátil en aorta abdominal y anormal por falta de pulsatilidad o rampa diastólica.

3. Hipertrofia ventricular izquierda: Definido por Índice de masa ventricular izquierda (IMVI) $>95 \mathrm{gr} / \mathrm{m}^{2}$ en mujeres $\mathrm{y}>115 \mathrm{gr} / \mathrm{m}^{2}$ en varones o por masa/talla ${ }^{2,7}>51 \mathrm{gr}$. o en pacientes con talla menor de 1,40 metros, la fórmula de masa/talla ${ }^{2,16}>45 \mathrm{gr}$. Para la medición de masa se utiliza la fórmula de Devereaux modificada: MVI (gr) $=0,8 \times\{1,04$ [(DdVI $\left.\left.+\mathrm{EPPd}+\mathrm{ESIVd})^{3}-(\mathrm{DdVI})^{3}\right]\right\}+0,6$. Las mediciones fueron realizadas en el corte de eje corto, vista paraesternal izquierda.

4. Función ventricular sistólica por fracción de acortamiento (FA): Definido normal: por FA > 30\% y anormal cuando la misma fue $<30 \%$

\section{Aspecto estadístico}

El presente es un estudio descriptivo. Se utilizaron para la descripción de las variables en estudio números absolutos para las variables categóricas, y promedio con desvío estándar para variables numéricas que ajustaron a normalidad. Todos los valores se expresaron con su intervalo de confianza del 95\% (IC95\%). El procesamiento de datos se realizó con SPSS 20.0

Tamaño de muestra: se incluyeron todos los registros clínicos de pacientes que cumplieron con los criterios de inclusión y ninguno de exclusión durante el período estimado.

\section{Reparos éticos}

Aprobado por el Comité de Docencia e Investigación y Comité de Ética e Investigación del Hospital General de Niños Pedro de Elizalde (nro143/18).

\section{Resultados}

Se incluyeron 33 pacientes, 26 varones. La edad promedio $10,2 \pm 3,8$ años. Sobre la antropometría, 21 peso normal, 7 sobrepeso (IMC pc 85-95) y 5 obesidad (IMC> pc95). 
La población fue heterogénea, con abordaje de la COAO por cirugía cardiovascular en $14 \mathrm{p}$; angioplastias sobre coartación nativa en $3 p$; angioplastias con stent en 10p; y seguimiento clínico en $6 p$.

El tiempo de seguimiento desde el abordaje es corto (media 5, 9 años; rango de 1 a 18 años).
Por PAc, $22 \mathrm{p}$ estaban normotensos, 8 hipertensos controlados, 2 prehipertensos, 1 hipertenso. Recibían medicación antihipertensiva 9p. (1 p prehipertenso y 8 hipertensos controlados (Tabla 1).

Tabla $N^{\circ}$ 1: Diagnóstico inicial por PA de consultorio y cambios de recategorización de los pacientes después del MAPA.

\begin{tabular}{|c|c|c|c|c|}
\hline & \multicolumn{2}{|c|}{ Presión arterial en consultorio } & \multicolumn{2}{|c|}{ Recategorización de la PA (MAPA) } \\
\hline & Diagnóstico & $\begin{array}{l}\text { Tratamiento } \\
\text { farmacológico }\end{array}$ & Diagnóstico & $\begin{array}{l}\text { Tratamiento } \\
\text { farmacológico }\end{array}$ \\
\hline 1 & NT & No & NT & No \\
\hline 2 & NT & No & NT & No \\
\hline 3 & NT & No & NT & No \\
\hline 4 & NT & No & NT & No \\
\hline 5 & NT & No & NT & No \\
\hline 6 & NT & No & NT & No \\
\hline 7 & NT & No & NT & No \\
\hline 8 & NT & No & NT & No \\
\hline 9 & NT & No & NT & No \\
\hline 10 & NT & No & NT & No \\
\hline 11 & NT & No & NT & No \\
\hline 12 & NT & No & NO VALORABLE & \\
\hline 13 & NT & No & PREHTA & No \\
\hline 14 & NT & No & PREHTA & No \\
\hline 15 & NT & No & PREHTA & No \\
\hline 16 & NT & No & PREHTA & No \\
\hline 17 & NT & No & PREHTA & No \\
\hline 18 & NT & No & PREHTA & No \\
\hline 19 & NT & No & $\mathrm{HE}$ & ENALAPRIL \\
\hline 20 & NT & No & $\mathrm{HE}$ & ENALAPRIL \\
\hline 21 & NT & No & $\mathrm{HE}$ & ENALAPRIL \\
\hline 22 & NT & No & $\mathrm{HE}$ & ENALAPRIL \\
\hline 23 & PREHTA no medicado & No & PREHTA & No \\
\hline 24 & PREHTA medicado & ATENOLOL & HTA C & Sin cambios \\
\hline 25 & HTA C & ENALAPRIL C/24hs & HTA NOCT & ENALAPRIL C/12hs \\
\hline 26 & HTA C & ENALAPRIL C/24 hs & HTA NOCT & ENALAPRIL C/12 hs \\
\hline 27 & HTA C & $\begin{array}{l}\text { ENALAPRIL } \\
\text { ATENOLOL }\end{array}$ & HTA NOCT & CRONOTERAPIA \\
\hline 28 & HTA C & ENALAPRIL & HTA C & Sin cambios \\
\hline 29 & HTA C & ENALAPRIL & HTA C & Sin cambios \\
\hline 30 & HTA C & ENALAPRIL C/24hs & HTA NC & $\begin{array}{l}\text { ENALAPRIL c/12 hs y } \\
\text { aumento dosis }\end{array}$ \\
\hline 31 & HTA C & ENALAPRIL & HTA NC & ENALAPRIL > DOSIS \\
\hline 32 & HTA C & $\begin{array}{l}\text { ENALAPRIL } \\
\text { ATENOLOL }\end{array}$ & HTA NC & Cronoterapia y ajuste dosis \\
\hline 33 & HTA & No & HTA & ENALAPRIL \\
\hline
\end{tabular}

Se obtuvieron registros completos de MAPA en 32 de los 33 estudios realizados, con un tiempo medio de registro de 23,3 horas y más de $70 \%$ de lecturas correctas. Se valoraron 11 pacientes normotensos, 7 prehipertensos, 3 pacientes con HTA nocturna, $4 \mathrm{HE}, 3$ con HTA controlada,
3 con HTA no controlada y 1 HTA. (Tabla 1) Fueron recategorizados 16 pacientes.

El Ecocardiograma doppler mostró un gradiente pico promedio en aorta descendente de $23 \mathrm{mmHg}$ (rango 13 a $40 \mathrm{mmHg}$ ). Se detectó rampa diastólica en aorta abdominal 
en 2 pacientes con re-COAO. Se describió IMVI normal en 29 pacientes e $\mathrm{HVI}$ en 4 pacientes. Al analizar los pacientes con $\mathrm{HVI}$, fueron normotensos en consultorio y solo en 2 se diagnosticó MAPA patológico (HTA nocturna y prehipertensión). Todos los pacientes tuvieron función sistólica ventricular izquierda normal.

Tratamiento antihipertensivo: De 33 pacientes, 9 estaban medicados por HTA. Luego del MAPA, 16 pacientes debieron recibir medicación y se ajustó la dosis y cronoterapia según resultados. Se reforzaron medidas higiénicodietéticas y en pacientes con preHTA se indicó control estricto y cercano. (3 meses) (Tabla 1).

\section{Discusión}

Se observaron cambios el manejo y diagnóstico luego de realizar MAPA en pacientes con COAO. La recategorización por MAPA detectó un $48 \%$ más de pacientes con aumento de presión, casi la mitad de pacientes que habían sido identificados como NT o HTA controlados modificaron este diagnóstico por otro fenotipo de HTA. (HE; pre HTA; HTA nocturna, HTA no controlada)

El estudio COALA mostró que un $25 \%$ de los pacientes con COAO recibe medicación antihipertensiva inicialmente ${ }^{(2)}$. En nuestra muestra, $27 \%$ de los pacientes recibían medicación antihipertensiva. El requerimiento de medicación aumentó de un $25 \%$ a un $53 \%$ en el estudio COAFU ${ }^{(3)}$. Esto implica la necesidad del correcto seguimiento de estos pacientes y esperar que una proporción mayor de pacientes puedan estar hipertensos en el futuro.

En nuestra descripción, 4 de 32 pacientes tuvieron diagnóstico de HE. Sin embargo, la HE ha sido reportada en el $45 \%$ de niños y adolescentes con COAO reparada ${ }^{(12)}$. Esta diferencia probablemente se explique porque nuestra población incluyó niños de menor edad y el desarrollo de la HTA tiene un carácter progresivo.

Nuestra descripción no estudió factores de riesgo. Muchos factores han sido involucrados en la fisiopatología de la HTA: técnica quirúrgica ${ }^{(2)}$; forma del arco gótico ${ }^{(4)}$; mecanismo de desajuste del acople ventriculoarterial ${ }^{(5)}$; edad al momento de la corrección ${ }^{(6-7)}$; hiperrespuesta del sistema renina angiotensina; disfunción de barorreceptores e hiperactividad del sistema nervioso simpático ${ }^{(12)}$.

EI MAPA es un auxiliar diagnóstico fundamental e indispensable en el seguimiento de $p$ con COAO, siendo una indicación específica. Las mediciones de PA en consultorio resultan insuficientes y el MAPA es el patrón de oro $^{(12)}$.

\section{Limitaciones del estudio:}

La población de nuestro estudio es pequeña, aunque incluye todos los $p$ con COAO del servicio que hubieran realizado el MAPA. Se realizó el análisis descriptivo de la población en forma consistente. El tiempo de seguimiento de los pacientes desde el abordaje es corto.

\section{Conclusiones}

EI MAPA permitió recategorizar la PA a los pacientes en el seguimiento con COAO, agregando los diagnósticos de HTA nocturna e HE y detectó HTA no controlada. Se transforma entonces en una herramienta indispensable en el seguimiento de estos pacientes.
Limitaciones de responsabilidad:

La responsabilidad de esta publicación es de los autores.

Fuentes de apoyo:

No hemos tenido fuentes de financiación externa.

\section{Originalidad:}

El trabajo remitido no ha sido publicado en ningún medio y no será enviado a otra revista científica o a cualquier otra forma de publicación, mientras dure la evaluación.

Cesión de derechos:

En el caso que el artículo sea publicado, todos los autores cederemos los derechos de autor.

Conflicto de interés:

Todos los autores manifestamos que no hubo conflicto de intereses.

\section{Bibliografía}

1. Nakamura K, Stefanescu Schmidt A. Treatment of Hypertension in Coarctation of the Aorta. Curr Treat Options Cardiovasc Med. 2016 Jun;18(6):40. doi: 10.1007/s11936-016-0462-x.

2. Hager A, Kanz S, Kaemmerer $H$, Schreiber C, Hess J. Coarctation Long-term Assessment (COALA): significance of arterial hypertension in a cohort of 404 patients up to 27 years after surgical repair of isolated coarctation of the aorta, even in the absence of restenosis and prosthetic material. $J$ Thorac Cardiovasc Surg. 2007 Sep;134(3):738-45. doi: 10.1016/j.jtcvs. 2007.04.027.

3. Bambul Heck P, Pabst von Ohain J, Kaemmerer H, Ewert $P$, Hager A. Arterial Hypertension after Coarctation-Repair in Longterm Follow-up (CoAFU): Predictive Value of Clinical Variables. Int J Cardiol. 2017 Nov 1;246:42-45. doi: 10.1016/j.ijcard.2017.05.084.

4. DeCampli WM. The good news is, we repaired your baby's coarctation.... J Thorac Cardiovasc Surg. 2017 Feb;153(2):415417. doi: 10.1016/j.jtcvs.2016.09.067.

5. Bruse JL, Khushnood A, McLeod K, Biglino G, Sermesant M, Pennec $X$, Taylor AM, Hsia TY, Schievano S; Modeling of Congenital Hearts Alliance Collaborative Group. How successful is successful? Aortic arch shape after successful aortic coarctation repair correlates with left ventricular function. J Thorac Cardiovasc Surg. 2017 Feb;153(2):418-427. doi: 10.1016/j.jtcvs.2016.09.018.

6. Lillitos PJ, Nassar MS, Tibby SM, Simmonds J, Salih C, Austin C, Anderson D, Krasemann $T$. Is the medical treatment for arterial hypertension after primary aortic coarctation repair related to age at surgery? A retrospective cohort study. Cardiol Young. 2017 Nov;27(9):1701-1707. doi: 10.1017/S1047951117001019.

7. Brown ML, Burkhart HM, Connolly HM, Dearani JA, Cetta F, Li Z, Oliver WC, Warnes CA, Schaff HV. Coarctation of the aorta: lifelong surveillance is mandatory following surgical repair. J Am Coll Cardiol. 2013 Sep 10;62(11):1020-5. doi: 10.1016/j.jacc.2013.06.016.

8. Simsolo R, Grunfeld B, Gimenez M, Lopez M, Berri G, Becú L, Barontini M. Long-term systemic hypertension in children after successful repair of coarctation of the aorta. Am Heart J. 1988 Jun;115(6):1268-73. doi: 10.1016/0002-8703(88)90020-8.

9. Lurbe E, Agabiti-Rosei E, Cruickshank JK, Dominiczak A, Erdine $S$, Hirth A, Invitti C, Litwin M, Mancia G, Pall D, Rascher W, Redon J, Schaefer F, Seeman T, Sinha M, Stabouli S, Webb NJ, Wühl E, Zanchetti A. 2016 European Society of Hypertension guidelines for the management of high blood pressure in children and adolescents. J Hypertens. 2016 Oct;34(10):1887-920. doi: 10.1097/HJH.0000000000001039.

10. Flynn JT, Kaelber DC, Baker-Smith CM, Blowey D, Carroll AE, Daniels SR, de Ferranti SD, Dionne JM, Falkner B, Flinn SK, Gidding SS, Goodwin C, Leu MG, Powers ME, Rea C, Samuels J, Simasek M, Thaker VV, Urbina EM; SUBCOMMITTEE ON SCREENING AND MANAGEMENT OF HIGH BLOOD PRESSURE IN CHILDREN. Clinical Practice Guideline for Screening and Management of High Blood Pressure in Children and Adolescents. Pediatrics. 2017 Sep;140(3):e20171904. doi: 
10.1542/peds.2017-1904. Erratum in: Pediatrics. 2017 Nov 30; Erratum in: Pediatrics. 2018 Sep;142(3).

11. Alpert BS, Bain HH, Balfe JW, Kidd BS, Olley PM. Role of the renin-angiotensin-aldosterone system in hypertensive children with coarctation of the aorta. Am J Cardiol. 1979 Apr;43(4):828-34. doi: 10.1016/0002-9149(79)90085-7.

12. Di Salvo G, Castaldi B, Baldini L, Gala S, del Gaizo F, D'Andrea A, Limongelli G, D'Aiello AF, Scognamiglio G, Sarubbi B, Pacileo $G$, Russo MG, Calabrò R. Masked hypertension in young patients after successful aortic coarctation repair: impact on left ventricular geometry and function. J Hum Hypertens. 2011 Dec;25(12):73945. doi: 10.1038/jhh.2010.118.

13. Lee MGY, Hemmes RA, Mynard J, Lambert E, Head GA, Cheung MMH, Konstantinov IE, Brizard CP, Lambert G, d'Udekem $Y$. Elevated sympathetic activity, endothelial dysfunction, and late hypertension after repair of coarctation of the aorta. Int $\mathrm{J}$ Cardiol. 2017 Sep 15;243:185-190. doi: 10.1016/j.ijcard.2017.05.075.

14. Cuspidi C, Sala C, Tadic M, Rescaldani M, Grassi G, Mancia G. Untreated Masked Hypertension and Subclinical Cardiac Damage: A Systematic Review and Meta-analysis. Am J Hypertens. 2015 Jun;28(6):806-13. doi: 10.1093/ajh/hpu231.

15. Lurbe E, Torro I, Alvarez V, Nawrot $T$, Paya R, Redon J, Staessen JA. Prevalence, persistence, and clinical significance of masked hypertension in youth. Hypertension. 2005 Apr;45(4):4938. doi: 10.1161/01.HYP.0000160320.39303.ab. 